\title{
Dosimetric evaluation employing TL and OSL techniques with different luminescent materials for clinical evaluation of extremity doses using electron beams applied to Total-Irradiation-of-Skin treatments
}

\author{
Shirlane Barbosa de Almeida \\ Radiation Metrology Center, Instituto de Pesquisas Energéticas e Nucleares-IPEN/CNEN \\ Avenida Prof. Lineu Prestes, 2242-Cidade Universitária \\ São Paulo, SP 05508-000, Brazil \\ sbipen@usp.br \\ Daniel Villani \\ Radiation Metrology Center, Instituto de Pesquisas Energéticas e Nucleares-IPEN/CNEN \\ Avenida Prof. Lineu Prestes, 2242-Cidade Universitária \\ São Paulo, SP 05508-000, Brazil \\ dvillani@ipen.br \\ Roberto Kenji Sakuraba \\ Radiation Therapy Department, Hospital Israelita Albert Einstein \\ Avenida Albert Einstein, 665-Morumbi \\ São Paulo, SP 05652-900, Brazil \\ rksakuraba@einstein.br \\ Ana Carolina Pires de Rezende \\ Radiation Therapy Department, Hospital Israelita Albert Einstein \\ Avenida Albert Einstein, 665-Morumbi \\ São Paulo, SP 05652-900, Brazil \\ ana.rezende@einstein.br \\ Silas Cardoso Santos \\ Radiation Metrology Center, Instituto de Pesquisas Energéticas e Nucleares-IPEN/CNEN \\ Avenida Prof. Lineu Prestes, 2242-Cidade Universitária \\ São Paulo, SP 05508-000, Brazil \\ silas.cardoso@usp.br \\ Letícia Lucente Campos \\ Radiation Metrology Center, Instituto de Pesquisas Energéticas e Nucleares-IPEN/CNEN \\ Avenida Prof. Lineu Prestes, 2242-Cidade Universitária \\ São Paulo, SP 05508-000, Brazil \\ lcrodri@ipen.br
}

Published 10 July 2018

This is an Open Access article published by World Scientific Publishing Company. It is distributed under the terms of the Creative Commons Attribution 4.0 (CC-BY) License. Further distribution of this work is permitted, provided the original work is properly cited. 
Total-skin electron beam (TSEB) irradiation is used to deliver a homogeneous dose distribution over the entire skin surface of a patient. TSEB dosimetry is quite complex as to the evaluation and measurement of absorbed dosage in the cutaneous region. This paper evaluates the performance of different dosimetric materials, using TL and OSL dosimetry, in the extremity-dose assessment of TSEB treatments using the six-dual-field technique and an anthropomorphic phantom. Dosimeters were selected with repeatability better than $\pm 5.0 \%$ and calibrated to $6-\mathrm{MeV}$ electron-beam dosimetry. Measurements were conducted in the abdominal region as a reference point and on the extremities. Results show expected deviations ranging up to $20 \%$ in the dose received in the extremities and good results in dose assessment using all dosimetric materials tested.

Keywords: Luminescence; treatment strategy applications; quality assurance in radiotherapy.

PACS numbers: 87.15.mq, 87.55.kh, 87.55.Qr

\section{Introduction}

One of the modalities of external radiation therapy is total-skin electron beam (TSEB) irradiation for delivering a homogeneous dose distribution over the entire skin surface of the patient. TSEB is the treatment of choice internationally for cutaneous T-cell lymphoma, both for curative and palliative purposes. In some anatomical regions, dosage may vary widely owing to the angle of treatment or the skin surface itself, which may be significantly curved and oblique to the plane of treatment. ${ }^{1,2}$

Because common external-radiation therapy-planning software does not cover TSEB irradiation, the commissioning and quality assurance of this application must be handled another way. The Hospital Israelita Albert Einstein follows the six-dual-field technique (also known as the Stanford technique) for the commissioning of TSEB treatments, as reported in Ref. 2 by the American Association of Physics in Medicine (AAPM). In this method, dual fields are created by varying the gantry rotation of the linear accelerator $\pm 17^{\circ}$ over the horizontal plane with reference to the waistline of the patient, creating a very large field over distance. Patients are treated in a two-day cycle with three dual fields per day - on the first day, in the anterior position and posterior right and left obliques, and on the second day, in the posterior position and anterior right and left obliques. The dual fields minimize x-ray contamination of the central axis and nonuniformity owing to the inverse-square-of-the distance law. ${ }^{2}$

Thermoluminescent dosimeters (TLDs), such as LiF:Mg,Ti TLD-100, CaSO 4 :Dy, and microdosimeters of LiF:Mg,Ti TLD-100 (with dimensions of $1 \times 1 \times 1 \mathrm{~mm}^{3}$ ), have demonstrated great efficiency in clinical electron-beam dosimetry ${ }^{3-7}$ and can be useful tools in detecting errors related to dose application. LiF:Mg,Ti is the TL material most used and studied in radiotherapy because of its near tissue equivalence and overall reliability. ${ }^{8}$ The Dosimetric Materials Laboratory of the Radiation Metrology Center/IPEN produces and markets $\mathrm{CaSO}_{4}$ :Dy as a powder and pellets (see Refs. 9-11). $\mathrm{CaSO}_{4}$ :Dy+Teflon pellets provide an extensive range of linearity to radiation, allowing a great variety of applications. ${ }^{11,12}$ Investigations in radiotherapy dose measurements have been recently performed..$^{5-7,13}$

Another type of material that has gained importance is aluminum oxide $\left(\mathrm{Al}_{2} \mathrm{O}_{3}: \mathrm{C}\right)$ used in optically stimulated luminescence (OSL) dosimeters. ${ }^{14,15}$ In general, OSL 
detectors have provided good results and are widely used in clinical dosimetry. ${ }^{12,14,16-19}$ The advantages of these dosimeters over TLDs include high sensitivity, faster readout, the possibility of multiple re-readings, and the obviation of heating treatments. ${ }^{15,16,20}$

The commissioning of the TSEB six-dual-field technique is experimentally described by Platoni et al. (see Ref. 21), as applied at Attikon University General Hospital. The authors used a parallel-plate ionization chamber and LiF:Mg,Ti TLD-100 dosimeters to validate treatment dosimetry. This paper evaluates the performance of different dosimetric materials using TL and OSL dosimetry in the extremity-dose assessment of TSEB treatments, using the six-dual-field technique and an anthropomorphic phantom.

\section{Material and Methods}

\subsection{Materials}

The dosimetric materials used in this study are shown in Fig. 1 and specified below:

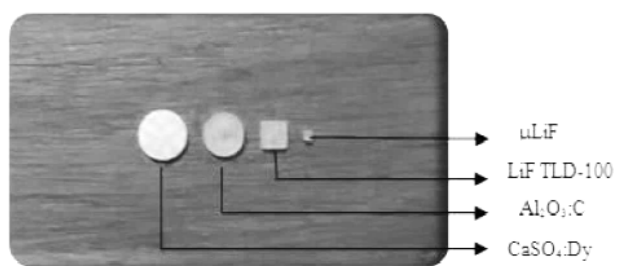

Fig. 1. The four luminescent dosimeters used in this study.

- $\mathrm{CaSO}_{4}$ :Dy+Teflon TLDs produced by IPEN, with $6 \mathrm{~mm}$ diameter, $0.8 \mathrm{~mm}$ thickness, and $50 \mathrm{mg}$ mass.

- $\quad$ LiF:Mg,Ti (TLD-100) TLDs produced by Thermo Scientific, with $3.15 \mathrm{~mm}$ side, 0.9 mm thickness, and $2 \mathrm{mg}$ mass.

- $\quad \mu \mathrm{LiF}: \mathrm{Mg}, \mathrm{Ti}$ (TLD-100) TLDs produced by Thermo Scientific, with $1.0 \mathrm{~mm}$ length, $1.0 \mathrm{~mm}$ thickness, and $1 \mathrm{mg}$ mass.

- $\mathrm{Al}_{2} \mathrm{O}_{3}: \mathrm{C}$ (TLD-500) used as OSLDs produced by Rexon TLD Systems \& Components Inc. (EUA) and supplied by the Brazilian company Pro-Rad, with $5 \mathrm{~mm}$ diameter, $0.9 \mathrm{~mm}$ thickness, and $72 \mathrm{mg}$ mass.

The dosimeters were divided into five groups to perform the measurements and one group for background dose control.

\subsection{Reading systems and bleaching treatments}

The TL measurements were performed using a Harshaw 4500 TLD reader in a nitrogen atmosphere. Both LiF:Mg,Ti dosimeters used a time-temperature profile (TTP) with preheating of $80^{\circ} \mathrm{C}$ and linear heating rate of $5^{\circ} \mathrm{C} / \mathrm{s}$ with a maximum temperature of $400^{\circ} \mathrm{C} .{ }^{8}$ For $\mathrm{CaSO}_{4}$ :Dy+Teflon pellets, a TTP with a linear heating rate of $10^{\circ} \mathrm{C} / \mathrm{s}$ with a 
maximum temperature of $300^{\circ} \mathrm{C}$ was used. ${ }^{8}$ Each reading cycle was performed within $\sim 40$ s.

The samples were thermally treated before and after irradiation. The LiF; $\mathrm{Mg}, \mathrm{Ti}$ detectors were annealed in a Vulcan 3-550 PD furnace at $400{ }^{\circ} \mathrm{C}$ for one hour, followed by rapid cooling to ambient temperature, then placed in a preheated Fanen 315-IEA 11200 surgical heater for two hours at $100{ }^{\circ} \mathrm{C}$. The CaSO4:Dy + Teflon dosimeters were annealed in a Vulcan 3-550 PD furnace at $300{ }^{\circ} \mathrm{C}$ for three hours. ${ }^{8,10}$

The OSL measurements of the TLD-500 were performed in a RIS $\varnothing$ TL/OSL-DA-20 reader equipped with the standard bialkali EMI 9235QB photomultiplier tube, a $90 \%$ intensity blue-LED light source used for OSL stimulation, and a Hoya U-340 filter of $7.5 \mathrm{~mm}$ thickness and $45 \mathrm{~mm}$ diameter. ${ }^{14}$ Each reading cycle was performed in $50 \mathrm{~s}$. Before and after each irradiation, the samples were optically annealed for $24 \mathrm{~h}$ using an Ourolux $1.3 \mathrm{~W}$ power lamp composed of 30 blue LEDs. The residual signal was evaluated after each bleaching cycle.

\subsection{Irradiation systems}

A $4 \pi$ geometry ${ }^{137} \mathrm{Cs}$ gamma irradiator (activity $=38.11 \mathrm{GBq}$ on 17 April 2014) from the Dosimetric Materials Laboratory of IPEN-LMD/IPEN was used to test the repeatability of all dosimeters used.

Clinical measurements were carried out using the Varian Clinac 23EX linear accelerator (manufactured by Varian Medical Systems, Inc., Palo Alto) at the radiotherapy center of the Hospital Israelita Albert Einstein (HIAE). For TSEB therapy, high dose-rate (HDR) total-skin electron mode (HDTSe) was selected, as well as the monitor units (MU) for dose delivery. The nominal energy of the electron beam produced is $6 \mathrm{MeV}$. The collimator was opened to $36 \times 36 \mathrm{~cm}$ after the insertion of a specific tray dedicated to TSEB practice.

\subsection{Dosimetric characterization}

The four types of dosimeters were characterized for the $6 \mathrm{MeV}$ energy electron beam from the Clinac 23EX. For each individual pellet, the individual calibration factor (i.e., signal/dose) was determined by its sensitivity to this radiation.

Irradiation with $150 \mathrm{MU}$ and $250 \mathrm{MU}$ (147.6 cGy and $246.0 \mathrm{cGy}$, respectively) was performed by positioning all dosimeters between two $0.3-\mathrm{cm}$ thick poly(methyl methacrylate) (PMMA) plates and at a depth of $1.30 \mathrm{~cm}$ obtained with a solid water bolus to put dosimeters under electronic equilibrium conditions. A field size of $20 \times 20 \mathrm{~cm}^{2}$ was used with a source-surface distance (SSD) of $100 \mathrm{~cm}$ and a $5-\mathrm{cm}$ solid water bolus for electron backscatter. These doses were chosen because of the documented linearity of response of all the dosimetric materials $8,10,14$ and the practical applicability to TSEB irradiation. The characterization setup is shown in Fig. 2. 

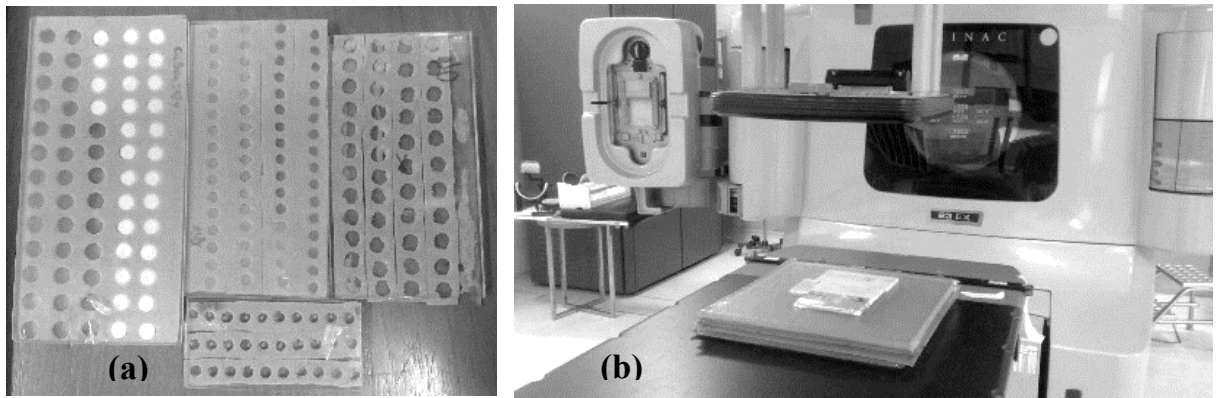

Fig. 2. Positioning the dosimeters to perform characterization. (a) Dosimeter placement between two PMMA plates and (b) irradiation setup in the Varian Clinac 23EX at HIAE.

\subsection{Experimental setup and irradiation}

The purpose of TSEB treatment is to distribute the dose as evenly as possible over the extent of the patient's skin surface. To obtain experimental data on absorbed doses in the extremities of patients, real conditions of TSEB treatment were simulated by using an Alderson Rando anthropomorphic phantom arranged on a turntable and a large $0.5 \mathrm{~cm}$ thick PMMA sheet to flatten the electron fields (Fig. 3).

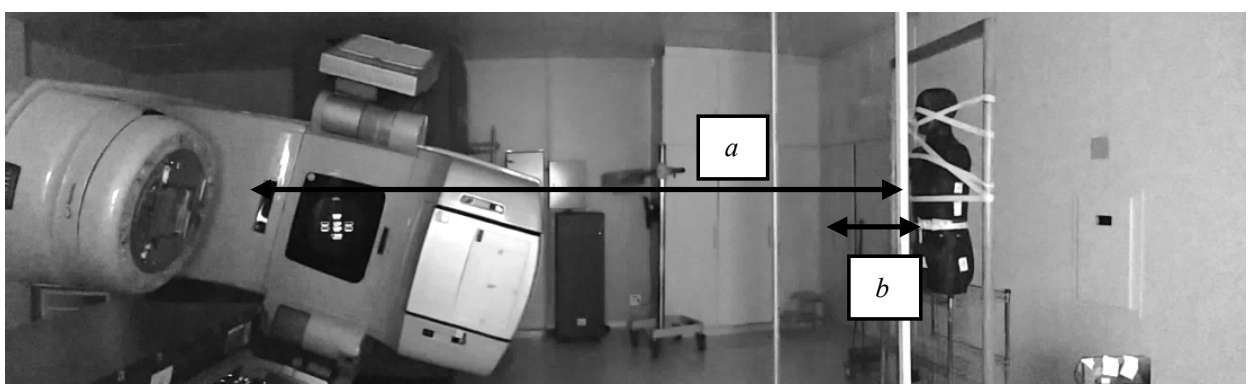

Fig. 3. TSEB experimental setup of irradiation using the Alderson Rando anthropomorphic phantom. Distance $a$, between the phantom and field isocenter, is $3 \mathrm{~m}$; distance $b$, between the phantom and the PMMA sheet, is $50 \mathrm{~cm}$.

The TL and OSL dosimeters were placed where anatomical extremities would be located and over the abdomen as a reference point $\left(\mathrm{Z}_{\mathrm{Ref}}\right){ }^{2,21}$ The measurements were performed on alternate days, as reported by AAPM, ${ }^{2}$ allowing greater study of sub- and over-dosage. The LiF:Mg,Ti TLD-100 dosimeters were used as reference dosimeters because of their composition properties, ${ }^{8}$ results for $6 \mathrm{MeV}$ electron-beam dosimetry, ${ }^{6,7}$ and similar validated TSEB applications. ${ }^{21}$

The experimental results of the absorbed doses are presented as the average of three dosimeter measurements, and the error bars are the standard deviation of the mean. All calculations were performed using Microsoft Excel 2016 software. Graphics were plotted using OriginPro 8.1 and measurements were expressed in cGy, as is commonly used by medical physicists in clinical applications. 


\section{Results}

\subsection{Dosimetric characterization}

The repeatability-test measurements were performed with a $4 \pi$ geometry ${ }^{137} \mathrm{Cs}$ gamma irradiator, in air and in electronic-equilibrium conditions, placing the pellets between two $0.3 \mathrm{~cm}$ thick PMMA plates. Irradiation to an absorbed dose of $5 \mathrm{mGy}$, readout, and bleaching were repeated five times to select samples with repeatability greater than $\pm 5 \%$. The standard deviation after the five readout cycles was $< \pm 4.0 \%$ for all selected samples.

The calibration factors for each sample were obtained using a $6 \mathrm{MeV}$ electron beam. They varied from $0.167 \pm 0.005 \mathrm{C} / \mathrm{cGy}$ for LiF:Mg,Ti TLD-100, 0.011 $\pm 0.001 \mathrm{C} / \mathrm{cGy}$ for $\mu \mathrm{LiF}: \mathrm{Mg}, \mathrm{Ti}$, and $0.054 \pm 0.002 \mathrm{C} / \mathrm{cGy}$ for $\mathrm{CaSO}_{4}$ :Dy to $6.03 \pm 1.07 \times 10^{3}$ counts/cGy for $\mathrm{Al}_{2} \mathrm{O}_{3}: \mathrm{C}$.

\subsection{Extremity TSEB-dosage assessment}

Many electrons that penetrate the skin surface are incident at large angles relative to the treatment plane, and the surface of the skin itself is often significantly curved and oblique to this plane. Consequently, the dose distribution on the skin may vary widely. In some regions, such as the thorax, posterior, right lateral, and right thigh, distribution varies only by a small percentage, as predicted by the dose distribution in the air. However, for many other parts of the body, dose may differ by more than $20 \%$ from abdominal readings $\left(\mathrm{z}_{\mathrm{Ref}}\right)$. Some areas, such as the axillae, scalp, palms, and medial thigh, receive at least $20 \%$ less dose than the reference point. ${ }^{2}$

In this treatment simulation, $470 \mathrm{MU}$ were selected to deliver $210 \mathrm{cGy}$ to the reference point (for calibration, see Refs. 2 and 21). Six other points were also measured: one on the right hand, two on the left hand, one on the right foot, and two on the left foot. The readout of all dosimeters was carried out after a two-day cycle of irradiation, as prescribed by the Stanford technique, giving the integrated dose at each point measured. Tables 1 to 4 present the experimental results obtained with LiF:Mg,Ti TLD-100, $\mu \mathrm{LiF}: \mathrm{Mg}, \mathrm{Ti}, \mathrm{CaSO}_{4}: \mathrm{Dy}$, and $\mathrm{Al}_{2} \mathrm{O}_{3}: \mathrm{C}$, respectively.

Table 1. Experimental results using LiF:Mg,Ti TLD-100 dosimeters.

\begin{tabular}{lcc}
\hline Position & Absorbed Dose (cGy) & $\begin{array}{c}\text { Percentage difference } \\
\text { relative to } \mathrm{Z}_{\text {Ref }}(\%)\end{array}$ \\
\hline Abdomen $\left(\mathrm{Z}_{\mathrm{Ref}}\right)$ & $205.0 \pm 1.0$ & 19.18 \\
Right Hand & $179.2 \pm 1.2$ & 17.87 \\
Left Hand 1 & $181.1 \pm 1.1$ & 11.95 \\
Left Hand 2 & $190.7 \pm 0.8$ & 24.66 \\
Right Foot & $266.6 \pm 1.2$ & 29.85 \\
Left Foot 1 & $277.3 \pm 3.2$ & 27.48 \\
Left Foot 2 & $272.2 \pm 1.3$ & \\
\hline
\end{tabular}


Table 2. Experimental results using $\mu \mathrm{LiF}: \mathrm{Mg}, \mathrm{Ti}$ dosimeters.

\begin{tabular}{lcc}
\hline Position & Absorbed Dose (cGy) & $\begin{array}{c}\text { Percentage difference } \\
\text { relative to TLD-100 }{ }^{\mathrm{a}}(\%)\end{array}$ \\
\hline Abdomen ( $\left.\mathrm{z}_{\text {Ref }}\right)$ & $221.8 \pm 0.8$ & 8.17 \\
Right Hand & $183.4 \pm 2.6$ & 2.38 \\
Left Hand 1 & $187.5 \pm 3.6$ & 6.50 \\
Left Hand 2 & $203.1 \pm 1.3$ & 3.52 \\
Right Foot & $275.1 \pm 7.2$ & 3.37 \\
Left Foot 1 & $272.8 \pm 4.9$ & 1.65 \\
Left Foot 2 & $288.7 \pm 5.2$ & 6.08 \\
\hline
\end{tabular}

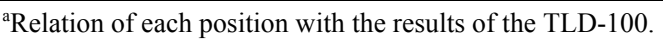

Table 3. Experimental results using $\mathrm{CaSO}_{4}: \mathrm{Dy}+$ Teflon dosimeters.

\begin{tabular}{lcc}
\hline Position & Absorbed Dose (cGy) & $\begin{array}{c}\text { Percentage difference } \\
\text { relative to TLD-100 }\end{array}$ \\
\hline Abdomen $\left(\mathrm{Z}_{\mathrm{Ref}}\right)$ & $205.0 \pm 1.7$ & 0.01 \\
Right Hand & $187.5 \pm 2.1$ & 4.63 \\
Left Hand 1 & $184.8 \pm 1.7$ & 2.05 \\
Left Hand 2 & $190.5 \pm 2.3$ & 0.13 \\
Right Foot & $277.2 \pm 3.6$ & 4.13 \\
Left Foot 1 & $287.2 \pm 3.4$ & 3.60 \\
Left Foot 2 & $245.1 \pm 2.4$ & 11.06 \\
\hline
\end{tabular}

${ }^{\text {a }}$ Relation of each position with the results of the TLD-100.

Table 4. Experimental results using $\mathrm{Al}_{2} \mathrm{O}_{3}$ :C OSL dosimeters.

\begin{tabular}{lcc}
\hline Position & Absorbed Dose (cGy) & $\begin{array}{c}\text { Percentage difference } \\
\text { relative to TLD-100 }(\%)\end{array}$ \\
\hline Abdomen $\left(\mathrm{z}_{\mathrm{Ref}}\right)$ & $188.8 \pm 13.7$ & 8.61 \\
Right Hand & $179.2 \pm 13.4$ & 0.02 \\
Left Hand 1 & $181.5 \pm 13.5$ & 0.20 \\
Left Hand 2 & $181.4 \pm 13.5$ & 0.10 \\
Right Foot & $277.5 \pm 16.7$ & 0.92 \\
Left Foot 1 & $269.6 \pm 16.4$ & 0.95 \\
Left Foot 2 & $188.8 \pm 13.7$ & 8.61 \\
\hline
\end{tabular}

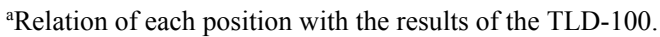


The agreement between the four dosimetric materials in each point of measurement can be readily observed in Fig. 4.

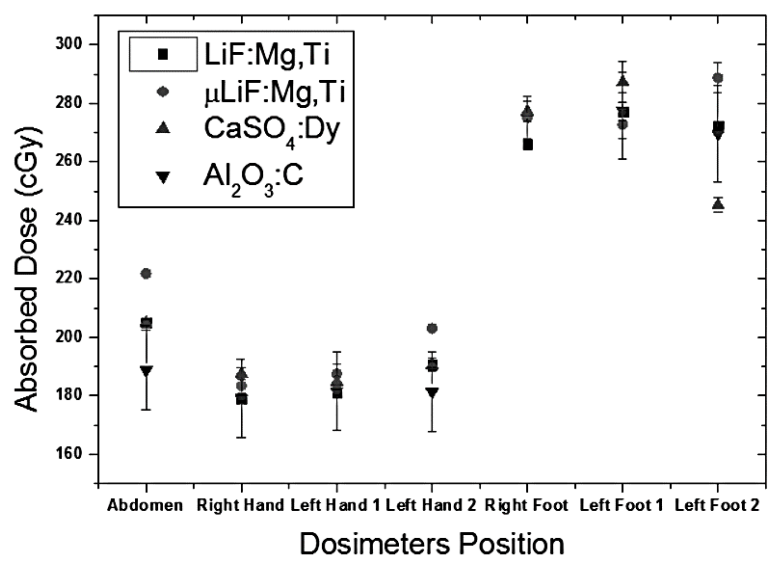

Fig. 4. Agreement between TL and OSL dosimeters measurements for each point studied.

\section{Discussion}

TSEB dosimetry is quite complex in the evaluation and measurement of absorbed dose in the cutaneous region. Solid-state luminescent (TL and OSL) dosimeters have demonstrated excellent results in assessing dose uniformity in the skin. The extremities are exposed in almost all treatment positions, resulting in a possibility of overdose that may vary up to $20 \%$ over the dose in the reference point. ${ }^{2}$ Similar results were obtained with the experimental dosimetry presented. These variations may be explained by errors in positioning, movement, and the positions of the dosimeters, since the Stanford technique is a two-day cycle.

It is found that for the abdominal region $\left(z_{\mathrm{Ref}}\right)$, agreement with the prescribed 210 cGy dose was $97.62 \%$ for $\mathrm{LiF}, \mathrm{Mg}$, Ti TLD-100, $94.38 \%$ for $\mu \mathrm{LiF}, \mathrm{Mg}, \mathrm{Ti}, 97.61 \%$ for $\mathrm{CaSO}_{4}: \mathrm{Dy}$, and $89.90 \%$ for $\mathrm{Al}_{2} \mathrm{O}_{3}: \mathrm{C}$. The results that best matched those obtained by the TLD-100 were the $\mathrm{CaSO}_{4}$ :Dy measurements, as indicated in Fig. 4. The results obtained with $\mu \mathrm{LiF}: \mathrm{Mg}$, Ti were slightly different from those with the TLD-100, even though they only differ by their dimensions. This can be explained by the lower TL intrinsic efficiency and reduced dimensions of $\mu \mathrm{LiF}: \mathrm{Mg}, \mathrm{Ti}^{7}{ }^{7}$ The $\mathrm{Al}_{2} \mathrm{O}_{3}: \mathrm{C}$ presented higher uncertainties; the percentage differences, however, remained acceptable.

\section{Conclusions}

The TL dosimeters showed good results in the assessment of extremity TSEB treatment doses, with percentage differences within the expected range. Variation in the results obtained for extremity doses can be explained by errors of positioning and movement of dosimeters. The $\mathrm{Al}_{2} \mathrm{O}_{3}$ : $\mathrm{C}$ OSL dosimeters presented higher uncertainties, but results were close to those of TLDs. 


\section{Acknowledgments}

The authors thank CNPq, CNEN, and FAPESP for their financial support and the radiation-therapy staff of the Hospital Israelita Albert Einstein for their assistance with electron irradiation.

\section{References}

1. R. A. Strohl, The role of total skin electron beam radiation therapy in the management of mycosis fungoides, Dermatol. Nurs. 6(3), 220 (1994).

2. C. J. Karzmack, J. Anderson, P. Fessenden, G. Svensson, A. Buffa, F. Khan et al., Total skin electron therapy: technique and dosimetry, AAPM Report No. 23, Report of Radiation Therapy Committee Task Group 30 (1987).

3. M. G. Nunes and L. L. Campos, Rad. Meas. 43(2), 459 (2008).

4. Luciana C. Matsushima et al., Response evaluation of $\mathrm{CaSO}_{4}: \mathrm{Dy}$; LiF:Mg,Ti and LiF:Mg, Ti microdosimeters using liquid water phantom for clinical photon beams dosimetry, https://www.ipen.br/biblioteca/2012/18533.pdf.

5. Luciana C. Matsushima et al., Revista Brasileira de Pesquisa e Desenvolvimento 13(1), 75 (2011).

6. A. Bravim et al., Rad. Meas. 46, 1979 (2011).

7. A. Bravim et al., Rad. Meas. 71, 315 (2014).

8. S. W. S. McKeever, M. Moscovitch and P. D. Townsend, Thermoluminescence Dosimetry Materials: Properties and Uses (Nuclear Technology Pub., Ashford, U.K., 1995).

9. L. L. Campos, J. Luminesc. 28(4), 481 (1983).

10. L. L. Campos and M. F. Lima, Rad. Prot. Dos. 14(4), 333 (1986).

11. L. L. Campos and M. F. Lima, Rad. Prot. Dos. 18(2), 95 (1987).

12. S. P. Morato et al., Nucl. Instrum. Meth. Phys. Res. 200(2-3), 449 (1982).

13. D. Villani et al., Rad. Meas. 106, 321 (2017).

14. S. W. S. McKeever, Nucl. Instrum. Meth. Phys. Res B. 184, 29 (2001).

15. E. G. Yukihara and S. W. S. McKeever, Optically Stimulated Luminescence: Fundamentals and Applications (Wiley, 2011).

16. M. S. Akselrod et al., Rad. Meas. 41, 78 (2007).

17. P. A. Jursinic, Med. Phys. 34(12), 4594 (2007).

18. R. M. Sanchez, E. Vano, J. M. Fernandez, M. Ginjaume and M. A. Duch, Radiat. Prot. Dosimetry 162(4), 569 (2014).

19. D. Opp, B. E. Nelms, G. Zhag, C. Stevens and V. Feygelman, J. Appl. Clin. Med. Phys. 14(4), 70 (2013).

20. S. W. S. McKeever and M. Moscovitch, Radiat. Prot. Dosimetry 104(3), 263 (2003).

21. K. Platoni et al., Physica Medica 28(2), 174 (2012). 